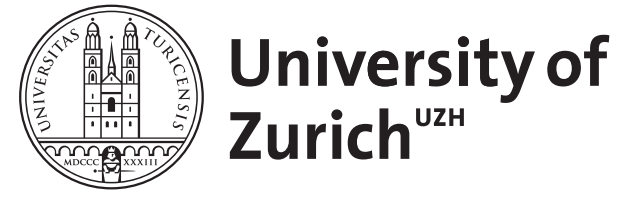

\title{
Cetuximab induced aseptic meningitis
}

Ulrich, A ; Weiler, S ; Weller, M ; Rordorf, T ; Tarnutzer, A A

\begin{abstract}
We report a 67-year-old man with recurrent advanced oropharyngeal squamous cell carcinoma who developed aseptic meningitis, with first symptoms arising approximately 9 hours after the first administration of cetuximab, and review the literature to identify key signs and symptoms of this condition. Cetuximab is a monoclonal antibody targeting the epidermal growth factor receptor which has been rarely associated with aseptic meningitis. Besides the case description, a MEDLINE search was performed. In five patients identified in the literature and our patient, the leading signs and symptoms included headache, neck stiffness and high fever developing within a few hours of the first cetuximab administration. Cerebrospinal fluid (CSF) analysis revealed severe pleocytosis (range: 528-2300/ l) with dominance of neutrophils ( $87 \%$ ). Clinical recovery within 1-2weeks was accompanied by normalization of CSF cell count within 4-7days. Re-challenge with cetuximab at a reduced dose caused recurrent aseptic meningitis in one of three patients. In summary, aseptic meningitis is a rare complication after first cetuximab exposure that the clinician should be aware of. CSF analysis is the key to diagnosis and recovery is usually complete within days to weeks after withdrawal of the drug. Re-challenge may be considered but bears the risk of recurrence.
\end{abstract}

DOI: https://doi.org/10.1016/j.jocn.2014.11.034

Posted at the Zurich Open Repository and Archive, University of Zurich ZORA URL: https://doi.org/10.5167/uzh-110464

Journal Article

Accepted Version

Originally published at:

Ulrich, A; Weiler, S; Weller, M; Rordorf, T; Tarnutzer, A A (2015). Cetuximab induced aseptic meningitis. Journal of Clinical Neuroscience, 22(6):1061-1063.

DOI: https://doi.org/10.1016/j.jocn.2014.11.034 


\section{Cetuximab induced aseptic meningitis - case report and review of the literature}

Ulrich A (1), Weiler S (2), Weller M (1), Rordorf T (3), Tarnutzer AA (1)

(1) Department of Neurology, University Hospital Zurich, Zurich, Switzerland

(2) Department of Clinical Pharmacology and Toxicology, University Hospital Zurich, Zurich, Switzerland

(3) Department of Oncology, University Hospital Zurich, Zurich, Switzerland

Short title: cetuximab induced aseptic meningitis

\section{Statistics:}

Word count of the abstract: 200

Word count of the text (excluding tables): 734

Character count of the title including spaces: 79

Number of tables: 2

\section{Corresponding author:}

Alexander A. Tarnutzer, MD. Department of Neurology, University Hospital Zurich,

Frauenklinikstr. 26, 8091 Zurich, Switzerland. Phone: +41 44255 5511, Fax: +41 44255

4380. Email: alexander.tarnutzer@access.uzh.ch

\section{Disclosures:}

Dr. Ulrich, Dr. Weiler, Dr. Rordorf and Dr. Tarnutzer do not report conflict of interest.

Prof. Weller has received research grants from Acceleron, Alpinia Institute, Bayer, Isarna, MSD, Merck Serono, PIQUR and Roche and honoraria for lectures or advisory board participation from Celldex, Isarna, Magforce, MSD, Merck Serono, Pfizer, Roche and Teva.

\section{Sources of funding:}

There are no specific sources of funding for this study

\section{Acknowledgments:}

We thank Prof. R. Stupp for critically reading the manuscript.

Key words:

Adverse drug reaction, aseptic meningitis, cetuximab, squamous cell carcinoma 


\begin{abstract}
Cetuximab is a monoclonal antibody targeting the epidermal growth factor receptor (EGFR), which has been rarely associated with aseptic meningitis. We report a case of aseptic meningitis with first symptoms about 9 hours after the first administration of cetuximab in a 67-year-old male with recurrent advanced oropharyngeal squamous cell carcinoma and review the literature to identify key signs and symptoms of this condition. Besides the case description a MEDLINE search was performed. In five patients identified in the literature and the patient presented here the leading signs and symptoms included headache, neck stiffness and high fever developing within few hours after first cetuximab administration. Cerebrospinal fluid (CSF) analysis revealed severe pleocytosis (range $=528-2300 / \mu 1$ ) with dominance of neutrophils ( $\geq 87 \%$ ). Clinical recovery within 1-2 weeks was accompanied by normalization of CSF cell count within 4 to 7 days. Re-challenge with cetuximab at a reduced dose caused recurrent aseptic meningitis in one of three patients. In summary, aseptic meningitis is a rare complication after first cetuximab exposure the clinician should be aware of. CSF analysis is the key and usually recovery is complete within days to weeks after withdrawal of the drug. Re-challenge may be considered but bears the risk of recurrence.
\end{abstract}


Cetuximab, a chimeric monoclonal antibody against the epidermal growth factor receptor (EGFR) has demonstrated activity as a single agent and in combination with chemotherapy or radiation in the treatment of metastatic or recurrent squamous cell cancer of head and neck, and in colorectal adenocarcinoma [1,2]. Aseptic meningitis has been observed as a rare complication of cetuximab administration.

Here we report aseptic meningitis after the first cetuximab administration $\left(400 \mathrm{mg} / \mathrm{m}^{2}\right)$ in a 67 year-old male treated for advanced oropharyngeal squamous cell carcinoma progressing on prior platinum-based treatment. Approximately nine hours after cetuximab infusion the patient complained of headache, showing neck stiffness, fever $\left(39.2^{\circ} \mathrm{C}\right)$ and psychomotor slowing. Cranial computed tomography revealed leucencephalopathy, but no other abnormalities. Cerebrospinal fluid (CSF) analysis (see Table 1) demonstrated an elevated cell count $(1413 / \mu 1$; normal range: $0-4 / \mu 1)$ with $92 \%$ neutrophil granulocytes, $8 \%$ macrophages and no cancer cells. Gram staining was negative. CSF protein was elevated (1.786 g/l; normal range $0.2-0.4 \mathrm{~g} / \mathrm{l})$, while glucose was normal $(3.5 \mathrm{mmol} / \mathrm{l}$, normal range 2.7 to $4.2 \mathrm{mmol} / \mathrm{l})$ and lactate was only slightly elevated $(3.0 \mathrm{mmol} / \mathrm{l}$, normal range 1.7 to $2.6 \mathrm{mmol} / \mathrm{l})$. Antibiotic and antiviral treatment was initiated with ceftazidim, vancomycin, amoxicillin and aciclovir plus dexamethasone. Treatment was stopped a few days later when PCR of neurotropic viruses (herpes simplex virus 1\&2, varicella-zoster virus, cytomegalovirus, Epstein-Barr virus), bacterial cultures/staining and fungal staining returned negative. We therefore interpreted the patient's condition as aseptic meningitis related to cetuximab. The patient gradually improved over the following days. Repeat CSF analysis eight days after admission (see Table 1) showed normalization of cell count $(1 / \mu \mathrm{l})$; while CSF protein was still elevated $(0.682 \mathrm{~g} / \mathrm{l})$. CSF glucose $(4.0 \mathrm{mmol} / \mathrm{l})$ and lactate $(1.5 \mathrm{mmol} / \mathrm{l})$ levels were normal and oligoclonal bands were negative. Another seven days later, CSF protein level had normalized, too $(0.359 \mathrm{~g} / \mathrm{l})$. The patient was discharged for rehabilitation four weeks after cetuximab treatment. Re-challenge with rituximab was not considered because of the reduced general condition due to tumor 
progression and comorbidities (end stage renal failure). The patient died six weeks later probably related to cardiac arrhythmias.

A MEDLINE-search ('cetuximab AND aseptic meningitis') identified three publications reporting on five patients (Table 2) [3-5]. Dose $\left(400 \mathrm{mg} / \mathrm{m}^{2}\right.$ ) and application (rate $=2 \mathrm{~h}$; first administration) of cetuximab were identical and symptom-onset was delayed by a few hours. Clinical presentation was similar with severe headaches, high fever and neck stiffness. CSF analysis revealed pleocytosis (range $=528-2300 / \mu \mathrm{l}$ ) with a dominance of neutrophil granulocytes $(\geq 87 \%)$. All patients recovered, usually within one week, as also reflected by normalization of CSF cell count. While re-challenge with cetuximab (with dose reduced to $250 \mathrm{mg} / \mathrm{m}^{2}$ ) was feasible in two cases [5], recurrent aseptic meningitis was reported in another case [4].

A search in the WHO Global Database of Individual Case Safety Reports (ICSR) ('cetuximab AND aseptic meningitis') identified 21 cases (2005-2014) from a total of 15.456 ICSRs involving cetuximab, but no further information about clinical presentation, recovery or CSF results was available.

Aseptic meningitis must be recognized as a rare and self-limiting adverse event that usually occurs within few hours after the first cetuximab administration. The combination of sudden headache, neck stiffness and high fever should prompt immediate CSF analysis including a broad search for bacterial, fungal or viral infections. Whereas the initial CSF inflammatory response is severe, a remarkably fast normalization of the CSF cell count accompanied by a rapid reduction of CSF protein is characteristic. Based on five published cases and 21 cases in the ICSR database, aseptic meningitis might be a very rare event after cetuximab treatment. The crossing of IgG over the blood-brain barrier might play a role in its pathogenesis [4]. Drug-induced meningitis as a diagnosis of exclusion was reported with other therapeutic 
antibodies too, most frequently with the application of intravenous immunoglobulins (IVIGs) $[6,7]$. Slowing the infusion rate, reducing the dose and premedication with steroids was proposed [5] in analogy to recommendations for the application of IVIGs [6, 7]. This approach was associated with lack of recurrence only in two of three cases.

In summary, because of its characteristic clinical presentation, tight temporal association to the first cetuximab application and negative CSF cultures/serologies, the clinician can identify this rare and self-limiting adverse event reliably. While antibiotic and virostatic treatment is initially advised, symptomatic treatment is sufficient as soon as CSF cultures/serologies return negative. Prognosis is usually good with complete recovery within 1-2 weeks. The decision for re-challenge with cetuximab must be made on an individual basis, but bears the risk of recurrence. 


\section{$\underline{\text { Tables }}$}

\begin{tabular}{|l|l|l|l|}
\hline \multicolumn{3}{|l|}{ Table 1: CSF- evolvement over time after application of cetuximab (=day 1) } \\
\hline parameter & day 1 & day 8 & day 15 \\
\hline $\begin{array}{l}\text { cell count } \\
\text { (normal range: } 0-4 / \mu \mathrm{l})\end{array}$ & 1413 & 1 & 1 \\
\hline neutrophils $(\%)$ & $92 \%$ & NA & NA \\
\hline $\begin{array}{l}\text { CSF protein } \\
\text { (normal range: } 0.2-0.4 \mathrm{~g} / \mathrm{l})\end{array}$ & 1.786 & 0.682 & 0.359 \\
\hline $\begin{array}{l}\text { glucose } \\
\text { (normal range } 2.7 \text { to } 4.2 \mathrm{mmol} / \mathrm{l})\end{array}$ & 3.5 & 4.0 & 2.8 \\
\hline $\begin{array}{l}\text { lactate } \\
\text { (normal range } 1.7 \text { to } 2.6 \mathrm{mmol} / \mathrm{l})\end{array}$ & 3.0 & 1.5 & 1.7 \\
\hline $\begin{array}{l}\text { PCR } \\
\text { (cytomegalovirus, Epstein-Barr } \\
\text { virus, herpes simplex virus 1\&2, } \\
\text { varicella-zoster virus) }\end{array}$ & negative & NA & NA \\
\hline $\begin{array}{l}\text { microbiology } \\
\text { (Cytosin- and periodic-acid- } \\
\text { Schiff-staining) }\end{array}$ & negative & NA & NA \\
\hline oligoclonal bands & NA & negative & NA \\
\hline cancer cells & negative & NA & NA \\
\hline
\end{tabular}

Abbreviations: $\mathrm{CSF}=$ cerebrospinal fluid; $\mathrm{NA}=$ not available; $\mathrm{PCR}=$ polymerase chain reaction 


\begin{tabular}{|c|c|c|c|c|c|c|c|c|}
\hline study & $\begin{array}{l}\text { case \#, } \\
\text { age, } \\
\text { gender }\end{array}$ & $\begin{array}{l}\text { type of cancer } \\
\text { / indication } \\
\text { for cetuximab }\end{array}$ & $\begin{array}{l}\text { cetuximab dose, } \\
\text { premedication }\end{array}$ & $\begin{array}{l}\text { symptoms and signs } \\
\text { (onset), imaging }\end{array}$ & $\begin{array}{l}\text { initial CSF analysis } \\
\text { (cell count, protein } \\
\text { level) }\end{array}$ & $\begin{array}{l}\text { follow-up CSF } \\
\text { analysis }\end{array}$ & treatment, recovery & re-challenge \\
\hline $\begin{array}{l}\text { Emani \& } \\
\text { Zaiden } \\
2012 \text { [4] }\end{array}$ & $\begin{array}{l}1 \\
54 \mathrm{y}, \mathrm{f}\end{array}$ & $\begin{array}{l}\text { squamous } \\
\text { maxillary } \\
\text { cancer (stage } \\
\text { IVb) }\end{array}$ & $\begin{array}{l}400 \mathrm{mg} / \mathrm{m}^{2} \text { (first } \\
\text { application), yes } \\
\text { (diphenhydramine } \\
\text { 50mg i.v.) }\end{array}$ & $\begin{array}{l}\text { frontal headache, } \\
\text { neck discomfort, } \\
39.9^{\circ} \mathrm{C} \text { fever (few } \\
\text { hours after } \\
\text { infusion), brain CT } \\
\text { (normal) }\end{array}$ & $\begin{array}{l}1025 / \mu 1 \text { with } 92 \% \\
\text { neutrophils, protein } \\
1.65 \mathrm{~g} / 1, \text { normal } \\
\text { glucose level, neg. } \\
\text { bacterial culture, } \\
\text { PCRs (HSV) neg. }\end{array}$ & NA & $\begin{array}{l}\text { empirical antibiotic } \\
\text { tx. Resolution of sx, } \\
\text { no complications. }\end{array}$ & $\begin{array}{l}\text { positive re-challenge after } 4 \\
\text { weeks }\left(250 \mathrm{mg} / \mathrm{m}^{2}\right) \text {, recurrent } \\
\text { CSF pleocytosis }(715 / \mu 1,93 \% \\
\text { neurophils), protein } 1.22 \mathrm{~g} / \mathrm{l}, \\
\text { premedication: } \\
\text { diphenhydramine, no steroids } \dagger\end{array}$ \\
\hline \multirow[t]{2}{*}{$\begin{array}{l}\text { Feinstein } \\
\text { et al. } \\
2009[5]\end{array}$} & $\begin{array}{l}2 \\
45 \mathrm{y}, \mathrm{m}\end{array}$ & $\begin{array}{l}\text { recurrent } \\
\text { laryngeal } \\
\text { squamous cell } \\
\text { carcinoma }\end{array}$ & $\begin{array}{l}400 \mathrm{mg} / \mathrm{m}^{2} \text { (first } \\
\text { application, } \\
\text { duration } 2 \mathrm{~h} \text { ), yes } \\
\text { (diphenhydramine } \\
\text { 50mg i.v.) }\end{array}$ & $\begin{array}{l}\text { frontal headache, } \\
38.9^{\circ} \mathrm{C} \text { fever (few } \\
\text { hours after } \\
\text { infusion), brain } \\
\text { imaging NR }\end{array}$ & $\begin{array}{l}2300 / \mu 1 \text { with } 98 \% \\
\text { neutrophils, protein } \\
1.04 \mathrm{~g} / 1, \text { normal } \\
\text { glucose level, neg. } \\
\text { cultures }\end{array}$ & $\begin{array}{l}\text { "resolution of } \\
\text { neutrophilic } \\
\text { pleocytosis", } \\
\text { normal protein } \\
\text { levels (day 4) }\end{array}$ & $\begin{array}{l}\text { empirical antibiotic } \\
\text { tx, aciclovir. No } \\
\text { information about } \\
\text { recovery }\end{array}$ & $\begin{array}{l}\text { negative re-challenge after } 1 \\
\text { week }\left(250 \mathrm{mg} / \mathrm{m}^{2} \text {, }\right. \\
\text { premedication: dexamethasone, } \\
\text { diphenhydramine }) \text { without } \\
\text { adverse events }\end{array}$ \\
\hline & $\begin{array}{l}3, \\
42 y, m\end{array}$ & $\begin{array}{l}\text { locally } \\
\text { advanced } \\
\text { squamous cell } \\
\text { carcinoma of } \\
\text { right tonsil }\end{array}$ & $\begin{array}{l}400 \mathrm{mg} / \mathrm{m}^{2} \text { (first } \\
\text { application, } \\
\text { duration } 2 \mathrm{~h} \text { ), yes } \\
\text { (diphenhydramine } \\
\text { 50mg i.v.) }\end{array}$ & $\begin{array}{l}\text { severe frontal } \\
\text { headache, } 39.4^{\circ} \mathrm{C} \\
\text { fever, neck } \\
\text { stiffness, } \\
\text { photophobia ( } \approx 8 \mathrm{~h} \\
\text { after infusion), brain } \\
\text { imaging NR }\end{array}$ & $\begin{array}{l}2267 / \mu 1 \text { with } 90 \% \\
\text { neutrophils, protein } \\
1.46 \mathrm{~g} / \mathrm{l}, \text { normal } \\
\text { glucose level, neg. } \\
\text { cultures }\end{array}$ & $\begin{array}{l}\text { "no white blood } \\
\text { cells", elevated } \\
\text { but improved } \\
\text { protein } \\
(69 \mathrm{mg} / \mathrm{dl})\end{array}$ & $\begin{array}{l}\text { empirical antibiotic } \\
\text { tx, acyclovir, } \\
\text { dexamethasone. } \\
\text { Recovery from } \\
\text { meningeal sx after } \\
12 \text { days }\end{array}$ & $\begin{array}{l}\text { negative re-challenge after } 2 \\
\text { weeks }\left(250 \mathrm{mg} / \mathrm{m}^{2} \text {, }\right. \\
\text { premedication: dexamethasone, } \\
\text { diphenhydramine, famotidine }) \\
\text { without adverse events }\end{array}$ \\
\hline \multirow[t]{2}{*}{$\begin{array}{l}\text { Nagovski } \\
\text { y et al. } \\
2010[3]\end{array}$} & $\begin{array}{l}4, \\
78 y, f\end{array}$ & $\begin{array}{l}\text { NSCLC (stage } \\
\text { IIIA) }\end{array}$ & $\begin{array}{l}400 \mathrm{mg} / \mathrm{m}^{2} \text { (first } \\
\text { application, } \\
\text { duration NR), NR }\end{array}$ & $\begin{array}{l}\text { severe headache, } \\
\text { nausea, vomiting, } \\
\text { neck stiffness (few } \\
\text { hours after } \\
\text { infusion), brain CT } \\
\text { (normal) }\end{array}$ & $\begin{array}{l}528 / \mu 1 \text { with } 87 \% \\
\text { neutrophils } \ddagger \text {, } \\
\text { "modestly elevated } \\
\text { protein”, normal } \\
\text { glucose levels }\end{array}$ & NA & $\begin{array}{l}\text { empirical antibiotic } \\
\text { tx (stopped after } \\
\text { infection was ruled } \\
\text { out). Recovery w/o } \\
\text { neurological } \\
\text { sequelae. }\end{array}$ & NR \\
\hline & $\begin{array}{l}5 \\
59 \mathrm{y}, \mathrm{m}\end{array}$ & $\begin{array}{l}\text { metastatic } \\
\text { NSCLC }\end{array}$ & $\begin{array}{l}400 \mathrm{mg} / \mathrm{m}^{2} \text { (first } \\
\text { application, } \\
\text { duration NR), NR }\end{array}$ & $\begin{array}{l}\text { acute } \\
\text { encephalopathy } \\
\text { (few hours after } \\
\text { infusion), brain CT } \\
\text { and MRI (normal) }\end{array}$ & $\begin{array}{l}\text { cell count and } \\
\text { fraction of } \\
\text { neutrophils NA } \S, \\
\text { protein } 1.16 \mathrm{~g} / \mathrm{l}, \\
\text { glucose } 2.8 \mathrm{mmol} / 1, \\
\text { neg. cultures }\end{array}$ & NA & $\begin{array}{l}\text { empirical antibiotic } \\
\text { tx (stopped after } \\
\text { infection was ruled } \\
\text { out). Recovery } \\
\text { "within several } \\
\text { days" }\end{array}$ & NR \\
\hline $\begin{array}{l}\text { Present } \\
\text { case }\end{array}$ & $\begin{array}{l}6 \\
67 \mathrm{y}, \mathrm{m}\end{array}$ & $\begin{array}{l}\text { recurrent } \\
\text { advanced } \\
\text { oropharyngeal } \\
\text { squamous cell } \\
\text { carcinoma }\end{array}$ & $\begin{array}{l}400 \mathrm{mg} / \mathrm{m}^{2} \text { (first } \\
\text { application, } \\
\text { duration } 2 \mathrm{~h} \text { ), yes } \\
\text { (clemastine } 2 \mathrm{mg} \\
\text { p.o.) }\end{array}$ & $\begin{array}{l}\text { headache, mutism, } \\
\text { hypertension, neck } \\
\text { stiffness, } 39.2^{\circ} \mathrm{C} \\
\text { fever }(\approx 9 \mathrm{~h} \text { after } \\
\text { infusion), brain CT } \\
\text { and MRI (non- } \\
\text { diagnostic) }\end{array}$ & $\begin{array}{l}1413 / \mu 1 \text { (normal } \\
\text { range } 0-4 / \mu 1) \text { with } \\
92 \% \text { neutrophils, } \\
\text { protein } 1.79 \mathrm{~g} / 1 \\
\text { (normal range } \\
<0.4), \text { normal } \\
\text { glucose level } \\
(3.5 \mathrm{mmol} / \mathrm{l}), \text { neg. } \\
\text { cultures / serologies }\end{array}$ & $\begin{array}{l}1 / \mu 1, \text { protein } \\
0.68 \mathrm{~g} / 1, \text { normal } \\
\text { glucose level } \\
(4.0 \mathrm{mmol} / \mathrm{l})\end{array}$ & $\begin{array}{l}\text { empirical antibiotic } \\
\text { tx, dexamethasone } \\
\text { (stopped after } \\
\text { infection was ruled } \\
\text { out). Myoclonic } \\
\text { jerks and NCSE } \\
\text { after } 3 \text { days. } \\
\text { Recovery within } 14 \\
\text { days. }\end{array}$ & $\begin{array}{l}\text { the patient and his family } \\
\text { decided against re-challenge of } \\
\text { cetuximab. The patient was } \\
\text { transferred to the hospice where } \\
\text { he died } 4 \text { weeks later. II }\end{array}$ \\
\hline
\end{tabular}


* We identified one additional case report with aseptic meningitis (dosage $100 \mathrm{mg} / \mathrm{m} 2$ ) from a phase 1 study [8]. With no further details available on this patient we did not include it in the table.

$\dagger$ Treatment with cetuximab was continued after the positive re-challenge, but no further adverse events occurred on exposures 3 and following.

$\$$ Based on the reported number of segmented polymorphonuclear leukocytes $(459 / \mu \mathrm{l})$, that made up $87 \%$ of all cells, a total CSF cell count of $528 / \mu 1$ was calculated.

$\S$ Nagovskiy and colleagues stated: „CSF evaluation showed 214 nucleated cells, 15 red blood cells, 81 segmented cells, and seven lymphocytes“ without providing further details such as total CSF cell count and whether these numbers were per $\mu$ l. Contacting the authors for clarification of the CSF cell count in this patient remained unsuccessful.

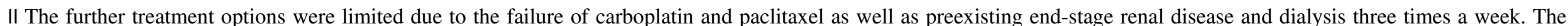

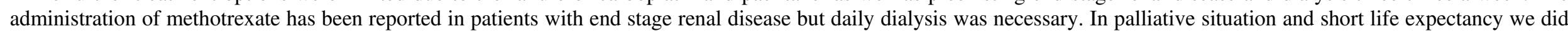
not consider this an option. Due to difficulties swallowing, the treatment with capecitabine was not possible and continuous infusion of 5 -fluorouracil during 5 days could not have been administrated because of dialysis.

Abbreviations: $\mathrm{CSF}=$ cerebrospinal fluid; $\mathrm{CT}=$ computed tomography; $\mathrm{HSV}=$ herpes simplex virus; $\mathrm{MRI}=$ magnetic resonance imaging; $\mathrm{NA}=$ not available; $\mathrm{NCSE}=$ non-convulsive status epilepticus; $\mathrm{NR}=$ not reported; NSCLC=non-small cell lung cancer; $\mathrm{PCR}=$ polymerase chain reaction. 


\section{$\underline{\text { References }}$}

[1] Blick SK, Scott LJ. Cetuximab: a review of its use in squamous cell carcinoma of the head and neck and metastatic colorectal cancer. Drugs. 2007;67:2585-607.

[2] Vermorken JB, Trigo J, Hitt R, Koralewski P, Diaz-Rubio E, Rolland F, et al. Open-label, uncontrolled, multicenter phase II study to evaluate the efficacy and toxicity of cetuximab as a single agent in patients with recurrent and/or metastatic squamous cell carcinoma of the head and neck who failed to respond to platinum-based therapy. J Clin Oncol. 2007;25:2171-7. [3] Nagovskiy N, Agarwal M, Allerton J. Cetuximab-induced aseptic meningitis. J Thorac Oncol. 2010;5:751

[4] Emani MK, Zaiden RA, Jr. Aseptic meningitis: a rare side effect of cetuximab therapy. J Oncol Pharm Pract. 2013;19:178-80.

[5] Feinstein TM, Gibson MK, Argiris A. Cetuximab-induced aseptic meningitis. Ann Oncol. 2009;20:1609-10.

[6] Hamrock DJ. Adverse events associated with intravenous immunoglobulin therapy. Int Immunopharmacol. 2006;6:535-42.

[7] Stiehm ER, Keller MA, Vyas GN. Preparation and use of therapeutic antibodies primarily of human origin. Biologicals. 2008;36:363-74.

[8] Baselga J, Pfister D, Cooper MR, Cohen R, Burtness B, Bos M, et al. Phase I studies of anti-epidermal growth factor receptor chimeric antibody C225 alone and in combination with cisplatin. J Clin Oncol. 2000;18:904-14. 